\title{
Effective real-time forecasting of inundation maps for early warning systems during typhoons
}

\author{
Jhih-Huang Wang ${ }^{1}$, Gwo-Fong Lin ${ }^{1, *}$, Bing-Chen Jhong ${ }^{1}$ \\ ${ }^{1}$ Department of Civil Engineering, National Taiwan University, Taipei 10617, Taiwan
}

\begin{abstract}
Accurate forecasts of hourly inundation depths are essential for inundation warning and mitigation during typhoons. In this paper, an effective forecasting model is proposed to yield 1- to 6-h leadtime inundation maps for early warning systems during typhoons. The proposed model based on Support Vector Machine (SVM) is composed of two modules, point forecasting and spatial expansion. In the first module, the rainfall intensity, inundation depth, cumulative rainfall and forecasted inundation depths are considered as model input for point forecasting. In the second module, the geographic information of inundation grids and the inundation forecasts of reference points are used to yield inundation maps for spatial expansion. The results show that the proposed model is able to provide accurate point forecasts at each inundation point. Moreover, the spatial expansion module is capable of producing accurate spatial inundation forecasts. Obviously, the proposed model provides reasonable spatial inundation forecasts, and is able to deal with the nonlinear relationships between inputs and desired output. In conclusion, the proposed model is suitable and useful for inundation forecasting.
\end{abstract}

\section{Introduction}

The heavy typhoon rainfall regularly result in serious disasters. Not only is typhoon rainfall an important and precious water resource, but also the flood inundation resulting from excessive rainfall brought by typhoons frequently leads to loss of human life and property. Therefore, more accurate inundation forecasts are always required. However, the inundation process is a highly nonlinear and complex physical process. Artificial neural networks (ANNs) are a kind of information processing system with great flexibility in modeling nonlinear systems. As to inundation forecasting, applications of NNs have been presented (Chang et al. [3]; Pan et al. [7]). The ASCE Task Committee $[1,2]$ and Maier and Dandy [6] have presented comprehensive reviews of the applications of ANNs in hydrology. To obtain effective forecasts of inundation, a model with good generalization ability is needed.

In recent years, support vector machines (SVMs) has been paid more and more attention and used for hydrological time series forecasting (Hong [4]; Lin et al. [5]). The algorithms and the architectures of the SVMs are guaranteed to be unique and globally optimal. Moreover, SVMs can be quickly trained with real-time data. However, because of only a few researches for applying SVMs on the forecasting of reg ional inundation maps. Therefore, the aforementioned advantages have prompted us to improve inundation forecasting by using SVM-based models.

To provide effective real-time forecasting of inundation maps for early warning system during typhoons. Therefore, the purpose of this paper is to propose a new forecasting technique, to yield 1- to 6-h lead time inundation maps for early warning system during typhoons. The proposed model based on Support Vector Machine (SVM) is composed of two modules, point forecasting and spatial expansion. In the first module, the rainfall intensity, inundation depth, cumulative rainfall and forecasted inundation depths are considered as model input for point forecasting. In the second module, the geographic information of inundation grids and the inundation forecasts of reference points are used to yield inundation maps for spatial expansion.

\section{Study area and materials}

An application to Chiayi City in southern Taiwan is conducted to demonstrate the superiority of the proposed model. Chiayi City is a major urban city in southwestern Taiwan with a population of about 270 thousand people, and has an area of approximately $60 \mathrm{~km}^{2}$. Inundation disasters have frequently been caused due to the topography characteristics and heavy typhoon rainfall in Chiayi City.

The hourly rainfall data were obtained from the Taiwan Water Resources Agency (TWRA). The inundation data are simulated and validated by a physically based two-dimensional model (FLO-2D), are used as a database for inundation forecasting in this paper. A total of 16 historical typhoon events were used to establish forecasting modules herein.

\footnotetext{
* Corresponding author: gflin@ntu.edu.tw
} 


\section{The proposed forecasting technology}

In this study, the proposed forecasting technology is composed of two steps: point forecasting step and spatial expansion step. The flowchart of the proposed forecasting methodology is shown in Fig. 1.

The first step is to establish the point forecasting module for each inundation point. The 1- to 6-h ahead forecasts of inundation depth are desired. The rain fall, cumulative rainfall and inundation depth are employed as inputs to develop the point forecasting module for each inundation point. The general form of the proposed point forecasting module (hereinafter SVM-A) can be expressed as:

$$
\hat{I}_{\mathrm{P}, t+\Delta t}=\left\{\begin{array}{c}
f_{\mathrm{SVM}}\left(R_{\mathrm{P}, t}, \ldots, R_{\mathrm{P}, t-\left(L_{\mathrm{R}}-1\right)}, C_{\mathrm{P}, t}, \ldots, C_{\mathrm{P}, t-\left(L_{C}-1\right)}, \ldots,\right. \\
\left.I_{\mathrm{P}, t}, \ldots, I_{\mathrm{P}, t-\left(L_{-}-1\right)}\right) \text { for } \Delta t=1 \\
f_{\mathrm{SVM}}\left(R_{\mathrm{P}, t}, \ldots, R_{\mathrm{P}, t-\left(\mathrm{L}_{\mathrm{R}}-1\right)}, C_{\mathrm{P}, t}, \ldots, C_{\mathrm{P}, t-\left(_{C}-1\right)}, \ldots,\right. \\
\left.I_{\mathrm{P}, t}, \ldots, I_{\mathrm{P}, t-\left(L_{-}-1\right)}, \hat{I}_{\mathrm{P}, t+1}, \ldots, \hat{I}_{\mathrm{P}, t+\Delta t-1}\right) \text { for } \Delta t>1
\end{array}\right.
$$

where $t$ is the current time, $\Delta t$ is the lead time period (from $1-$ to $6-\mathrm{h}$ ), $R_{\mathrm{P}, t}$ is the hourly rainfall of the inundation point at time $t, C_{\mathrm{P}, t}$ is the cumulative rainfall of the inundation point at time $t, I_{\mathrm{P}, t}$ is the inundation depth of the inundation point at time $t, \hat{I}_{\mathrm{P}, t+\Delta t}$ is the forecasted inundation depth of the inundation point at time $t+\Delta t, L_{R}$ denote the lag length of rainfall, $L_{C}$ denote the lag length of cumulative rainfall and inundation, $L_{I}$ denote the lag length of inundation. In addition, the SVM-A is compared with a conventional module (hereinafter SVM-B) to show the improvement in point forecasting step. The input of the conventional module are rainfall and inundation. The general form of the conventionalmodule can be expressed as:

$$
\hat{I}_{\mathrm{P}, t+\Delta t}=f_{\mathrm{SVM}}\left(R_{\mathrm{P}, t}, \ldots, R_{\mathrm{P}, t-\left(L_{R}-1\right)}, I_{\mathrm{P}, t}, \ldots, I_{\mathrm{P}, t-\left(L_{1}-1\right)}\right)
$$

The second step, the forecasted results from the point forecasting step are expanded to spatial estimation. First, the inundation data are classified by clustering technology. For each classification, the forecasted inundation depths in the point forecasting step, rainfall, cumulative rainfall and inundation depth are employed as inputs to develop the spatial expansion module. The general form of the spatial expansion module is:

$$
\hat{I}_{g, t+\Delta t}=f_{\mathrm{SVM}}\left(X_{g}, Y_{g}, H_{g}, R_{g, t}, C_{g, t}, \hat{I}_{\mathrm{P}, t+\Delta t}\right)
$$

Where $\hat{I}_{g, t+\Delta t}$ is the forecasted inundation depth of grid $g$ at time $t+\Delta t, X_{g}$ and $Y_{g}$ are the coordinates of grid $g$, $H_{g}$ is the elevation of grid $g, R_{g, t}$ is the rainfall of grid $g$ at time $t$ and $C_{g, t}$ is the cumulative rainfall of grid $g$ at time $t$. Once the inundation depths of all the inundated grids are forecasted, the inundation map is then obtained.

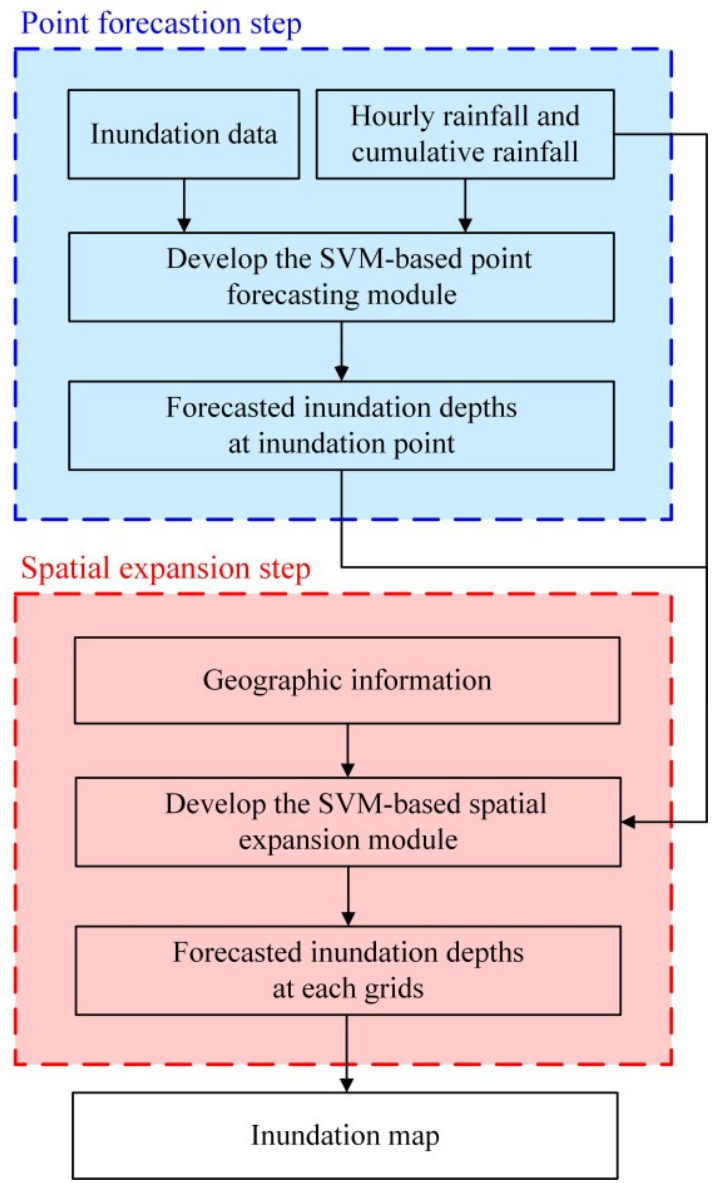

Fig. 1. Flowchart of the proposed forecasting technique.

\section{Results and discussion}

To demonstrate the improvement in forecasting performance using the proposed point forecasting module, we first focus on the comparison between the proposed module (SVM-A) and the conventional module (SVM-B). The performance of modules is evaluated by several criteria, such as root mean square error (RMSE) and correlation coefficient (CC). As shown in Fig. 2, the bar charts clearly show that the RMSE values of the SVM-A are lower than those of the SVM-B. Moreover, the RMSE values of the SVM-A increase more slowly than those of the SVM-B with increasing lead time. The negative impact of increasing forecast lead time has been effectively decreased by using the SVM-A. In addition, the CC values of the SVM-A are higher than those of the SVM-B. It is obvious that the performance of the SVM$B$ gets worse. The results confirm that the proposed module effectively improves the forecasting performance. The use of the proposed module instead of the conventional module effectively decreases the negative impact of increasing forecast lead time. 


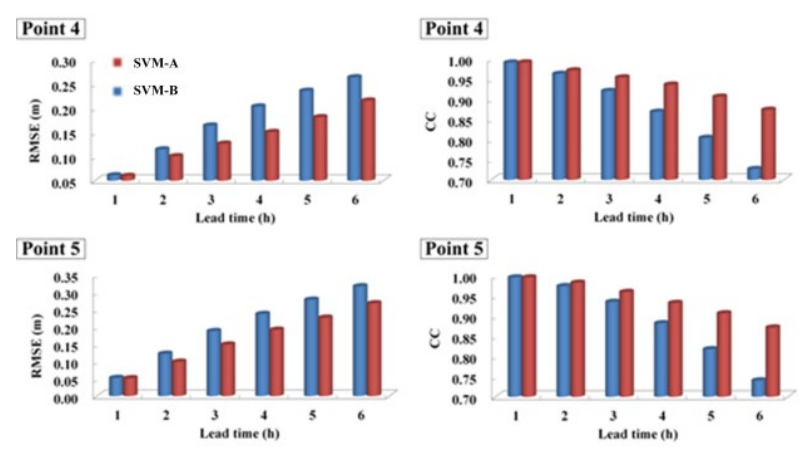

Fig. 2. RMSE and CC values of SVM-A and the SVM-B.

The comparison of the inundation data and corresponding forecasts resulting from the proposed technique for Typhoon Krosa is presented in Fig. 3. The forecasted results from the proposed technique are in good agreement with the inundation data, especially for the western and central region of the study area. Moreover, the pattern of the map obtained from the proposed technique is similar to that fro $m$ the inundation data for 1- to 6-h lead time.
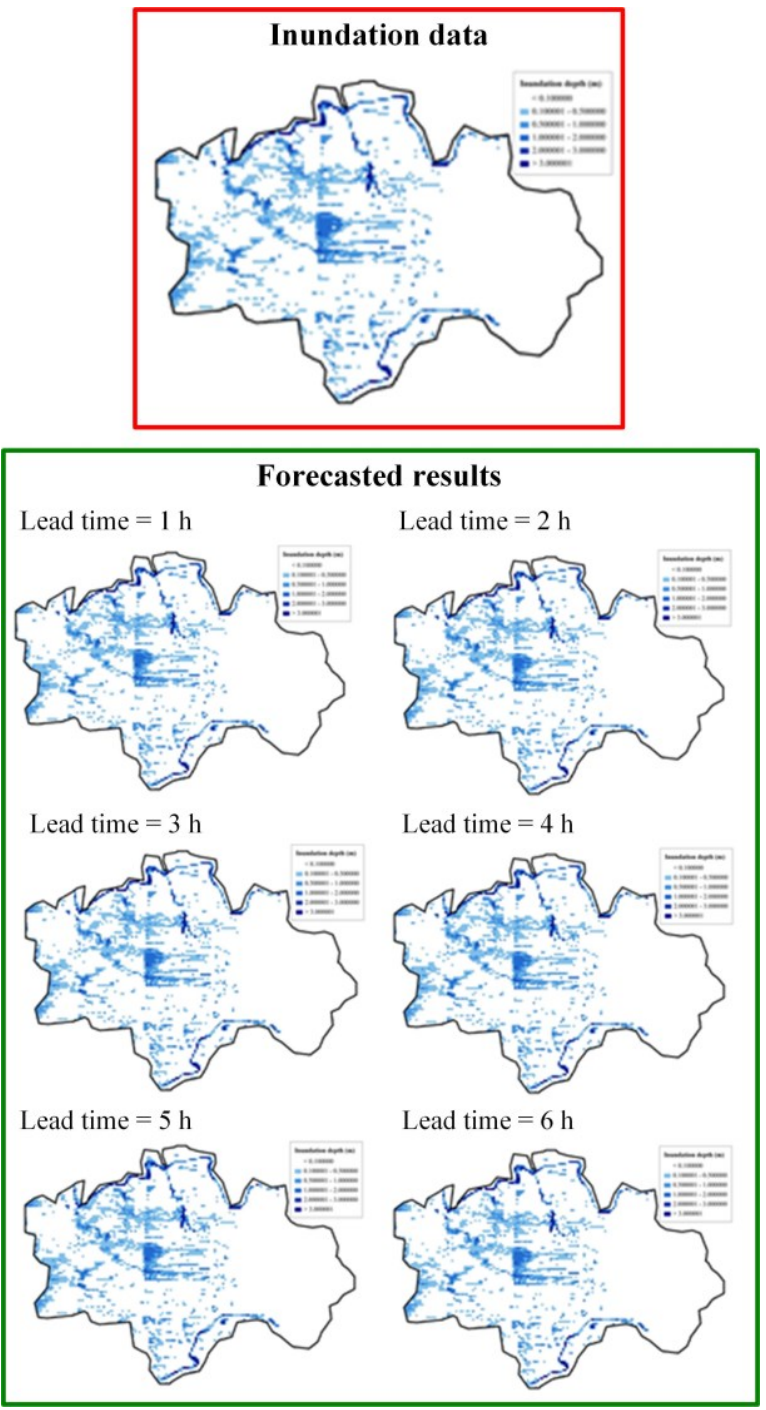

Fig. 3. Comparison of the inundation data and the forecasted results of the proposed model.

\section{Conclusions}

The purpose of this paper is to provide effective realtime forecasting of inundation maps for early warning system during typhoons. The results show that the proposed model is able to provide accurate point forecasts at each inundation point. Moreover, the spatial expansion module is capable of producing accurate spatial inundation forecasts. The maps of the forecasted results are similar to that of the inundation data. It is concluded that the proposed technique is suitable to be integrated with the decision support system. In the future, observation of inundation depth could be applied to the proposed forecasting approach to forecast inundation maps if the historical observed data of inundation was obtained from the 7-Eleven stores. The proposed forecasting approach is also expected to be useful to support inundation warning systems.

\section{References}

1. ASCE Task Committee on Application of Artificial Neural Networks in Hydrology, Artificial Neural Networks in hydrology I: Preliminary Concepts, Journal of Hydrologic Engineering 5, 115-123 (2000).

2. ASCE Task Committee on Application of Artificial Neural Networks in Hydrology, Artificial Neural Networks in hydrology II: Hydrological Applications, Journal of Hydrologic Engineering 5, 124-137 (2000).

3. L.C. Chang, H.Y. Shen, Y.F. Wang, J.Y. Huang, Y.T. Lin, Clustering-based hybrid inundation model for forecasting flood inundation depths, Journal of Hydrology 385, 257-268 (2010).

4. W.C. Hong, Rainfall Forecasting by Technological Machine Learning Models, Applied Mathematics and Computation 200,41-57 (2008).

5. G.F Lin, Y.C. Chou, M.C. Wu, Typhoon flood forecasting using integrated two-stage support vector machine approach, Journal of Hydrology $\mathbf{4 8 6}$, 334-342 (2013).

6. H.R. Maier, G.C. Dandy, Neural networks for the prediction and forecasting of water resources variables: A review of modeling issues and applications, Environmental Modelling and Software 15, 101-124 (2000).

7. T.Y. Pan, J.S. Lai, T.J. Chang, H.K. Chang, K.C. Chang, Y.C. Tan, Hybrid neural networks in rainfall-inundation forecasting based on a synthetic potential inundation database, Natural Hazards and Earth System Sciences 11, 771-787 (2011). 